\title{
Design of an Integrated Filterbank for DESHIMA: On-Chip Submillimeter Imaging Spectrograph Based on Superconducting Resonators
}

\author{
A. Endo • P. van der Werf • R.M.J. Janssen • \\ P.J. de Visser • T.M. Klapwijk · J.J.A. Baselmans • \\ L. Ferrari · A.M. Baryshev • S.J.C. Yates
}

Received: 17 July 2011 / Accepted: 5 January 2012 / Published online: 24 January 2012

(C) The Author(s) 2012. This article is published with open access at Springerlink.com

\begin{abstract}
An integrated filterbank (IFB) in combination with microwave kinetic inductance detectors (MKIDs), both based on superconducting resonators, could be used to make broadband submillimeter imaging spectrographs that are compact and flexible. In order to investigate the possibility of adopting an IFB configuration for

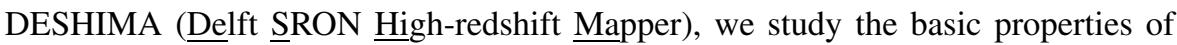
an IFB circuit using electromagnetic simulation. In an idealized situation, one could integrate onto a 4-inch wafer a 9 pixel $\times 920$ color 3 dimensional imaging device, which instantaneously covers multiple submillimeter telluric windows with a resolving power of $f / d f=1000$. We also simulate a slightly more realistic situation, in which the dimensions of the filters differ from their designed values, to see how fabrication errors introduce scattering in the transmission of the channels.
\end{abstract}

Keywords Kinetic inductance detector $\cdot$ Submillimeter wave $\cdot$ Spectrometer

A. Endo (凶) · R.M.J. Janssen · P.J. de Visser · T.M. Klapwijk

Kavli Institute of NanoScience, Faculty of Applied Sciences, Delft University of Technology, Lorentzweg 1, 2628 CJ Delft, The Netherlands

e-mail: A.Endo@tudelft.nl

P. van der Werf

Leiden Observatory, Leiden University, P.O. Box 9513, 2300 RA Leiden, The Netherlands

P.J. de Visser · J.J.A. Baselmans · S.J.C. Yates

SRON, Sorbonnelaan 2, 3584 CA Utrecht, The Netherlands

L. Ferrari · A.M. Baryshev

SRON, Landleven 12, 9747 AD Groningen, The Netherlands

A.M. Baryshev

Kapteyn Astronomical Institute, University of Groningen, P.O. Box 800, 9700 AV Groningen, The Netherlands 


\section{Introduction}

Submillimeter galaxies (SMGs) are massive star-forming galaxies found in the early Universe. The distribution of SMGs across a wide range of redshifts $z$ is of great importance for studies of the cosmic history of star- and galaxy-formation, and the evolution of the cosmic large scale structure [1]. While SMGs are expected to be found in vast quantities in the coming years, by virtue of large format $2 \mathrm{D}$ submillimeter imaging cameras, the measurement of the third dimension-redshift $z$ containing the history-would most likely become the rate-limiting step. An ideal instrument for quickly measuring the $z$ of SMGs would be a submillimeter direct detection spectrometer with a resolving power of $f / d f \sim 1000$, to match the typical width of the emission lines from SMGs [2] with rotation velocities of $\geq 300 \mathrm{~km} \mathrm{~s}^{-1}$. Although the first generation of these so-called $z$-machines has successfully detected lines from high redshift sources $[3,4]$, the instantaneous bandwidth and number of pixels are limited by both the number of detectors and the optics. Moreover, the spatial sampling has been limited to 0 or 1 dimensions.

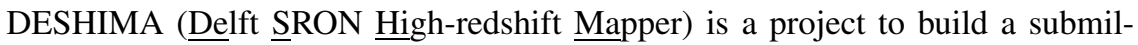
limeter imaging spectrograph using the advantage of multiplexability that microwave kinetic inductance detectors (MKIDs) have. The number of detectors of MKID cameras [5] are quickly exceeding 10,000. If these detectors are to be used for an imaging spectrograph, it is enough for ten or more pixels to instantaneously cover multiple submillimeter telluric windows with sufficient frequency resolution. Such an instrument would be ideal for submillimeter telescopes, such as the Atacama Pathfinder EXperiment (APEX), the Atacama Submillimeter Telescope Experiment (ASTE), and the Cerro Chajnantor Atacama Telescope (CCAT). However, it is challenging to build such a spectrometer using grating optics, from the perspective of size, complexity, and also to achieve a convenient spatial sampling.

One of the alternative frequency-selecting elements is a filterbank. Filterbanks have been commonly used at microwave frequencies, and it is known [6] that a pair of coupled coaxial cable resonators can be used as a band pass filter with an attractive resolving power of 1000. At submillimeter wavelengths, such a filterbank will scale down in size to where the entire filterbank will fit onto a single chip, together with the MKIDs. Such an integrated filterbank (IFB) could take advantage of the compactness, flexibility and mass-production capability that superconducting thin-film technology offers also to MKIDs. Independently from us, a similar concept was proposed by Kovacs and Zmudizinas (private communication).

In this study, we use numerical simulation to investigate the possibility of adopting an IFB design for DESHIMA. We will begin with a single filter, and then analyze multiple filters in a chain.

\section{Numerical Simulation of the Integrated Filterbank}

The integrated filterbank design considered in this study is depicted in Fig. 1(a, b). The circuit can consist of either coplanar waveguides (CPWs) or microstrip lines (MSLs), or a combination of both. CPWs have an advantage that it is easier to 

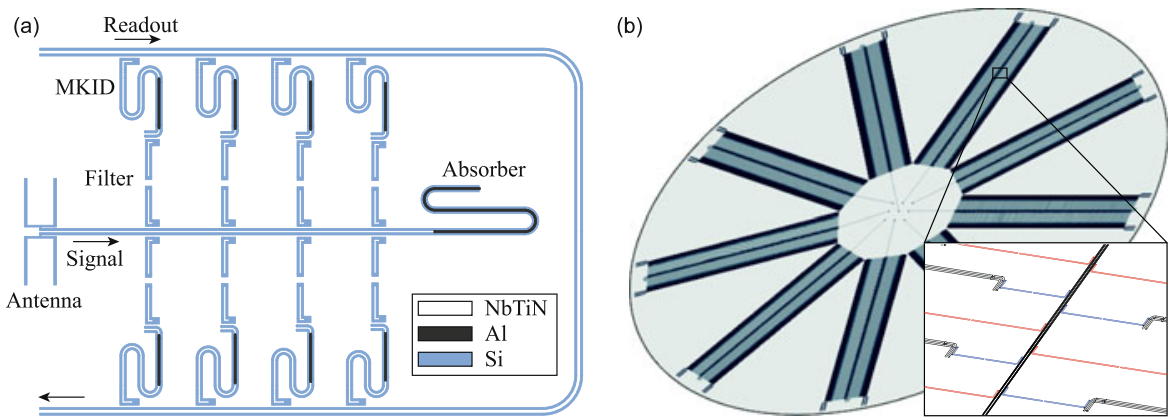

Fig. 1 (Color online) (a) Conceptual drawing of the integrated filterbank. The length scales are modified to enhance visibility. Each detection channel consists of a filter, made of a pair of low-loss resonators which are resonant at the signal wavelength $(\sim 100 \mu \mathrm{m})$, and an MKID, made of a lossy resonator which is resonant at the wavelength of the readout tone $(\sim 5 \mathrm{~mm})$. The length of the resonators is made slightly different from channel to channel. The low-loss section is made fully out of NbTiN (white), whereas the lossy section uses Al (black). The gray (blue in color) section indicates the region where there is no metal. At the end of the signal line is a matched absorber. (b) Example of a layout of DESHIMA on a 4-inch diameter wafer. There are 9 spiral antennas placed in the center with a nearest-neighbor pixel distance of $2 \mathrm{~mm}$. From each antenna stretches a transmission line towards the integrated filterbank. The filterbank is designed to cover $320-475 \mathrm{GHz}$ and $600-950 \mathrm{GHz}$ with a resolving power of $1000 \mathrm{using} 920$ channels per pixel. The readout signal can access the MKIDs from the bonding pads located near the edge of the wafer. The inset is a close-up view of the filters of some channels. The longer filters (red) for the low-frequency band are spaced $\lambda / 2$ away from each other, whereas the shorter filters (blue) for the higher frequency band are spaced $\lambda$ away from each other

make shorts to the ground plane. Moreover, lens-antenna coupled, NbTiN/Al hybrid MKIDs based on CPWs have demonstrated [7] photon noise limited sensitivity under loading powers as low as $100 \mathrm{fW}$ : The single-channel loading power expected for a typical observation using DESHIMA. On the other hand, the ground plane of an MSL could offer better stray light shielding, compared to a CPW which can couple to stray light through the parasitic slotline mode. The signal shining on the antenna will propagate along a transmission line (signal line, hereafter) to which multiple filters are coupled. Each channel of the filterbank consists of two sections; the first is a pair of low-loss resonators which act as a narrow bandpass filter, and the second is a lossy resonator which functions as an MKID. The MKID is a microwave resonator, coupled to the filter on one end and to the readout transmission line on the other end. At the end of the signal line is a long, lossy transmission line that absorbs the uncaptured signal to suppress standing waves. The low-loss section can be made of superconductors with gap frequencies higher than $1 \mathrm{THz}$, such as $\mathrm{NbTiN}$ or $\mathrm{NbN}$. $\mathrm{NbTiN}$ is also preferable for the MKID section because of its low two-level-system noise [8]. In the lossy sections, the stripline is replaced by a superconductor with a gap frequency lower than the signal, such as $\mathrm{Al}$ or TiN.

The integrated filterbank is modeled using a commercial 2.5 dimensional electromagnetic simulator Sonnet [9]. First we model a single filter by replacing the antenna, the absorber, and the connection from the shorted end to the rest of the MKID, by matched ports. The kinetic inductance of the NbTiN film is taken into account by assigning a surface inductance of $0.5 \mathrm{pH} / \mathrm{square}$, which is a typical measured value of our films [8] with a thickness of $300 \mathrm{~nm}$. The geometry of a single channel CPW filter 


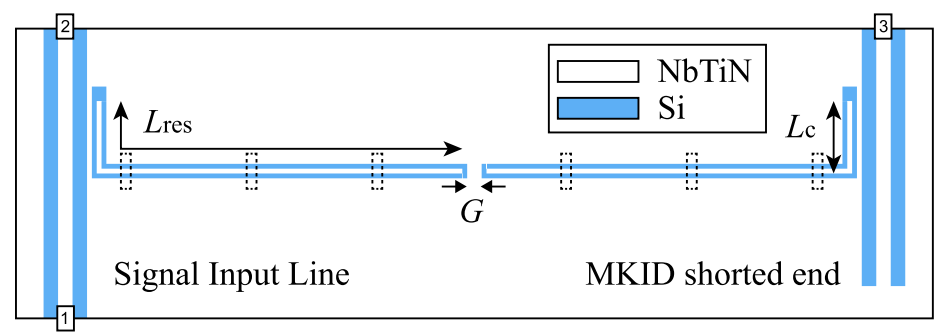

Fig. 2 (Color online) Example of a 3-port, 2.5 dimensional model based on coplanar waveguides (CPWs). The model consists of CPWs made of NbTiN (white) on $500 \mu \mathrm{m}$ of Si (gray, blue in color), suspended in vacuum. The vertical CPW on the left with ports 1 and 2 represents the line from the antenna carrying the signal. The vertical CPW on the right connected to port 3 represents the shorted end of a microwave kinetic inductance detector (MKID). The center strip and the slots of these two lines are all $3 \mu \mathrm{m}$ wide. In between the two lines is the filter, which consists of a pair of half wavelength resonators. The CPW for the filter has a center strip and slots which are all $1 \mu \mathrm{m}$ wide. The length of the entire resonator, the coupler, and the width of the ground plane between the two resonators are defined as $L_{\mathrm{res}}, L_{\mathrm{c}}$, and $G$, respectively. The width of the ground plane in between the filters, and the signal line or the MKID, is kept at $2 \mu \mathrm{m}$. The dotted rectangles represent airbridges made of a perfect electric conductor

is shown in Fig. 2. An equivalent circuit can be built using MSLs as well. As seen in Fig. 3(a), the use of two coupled halfwave resonators adds a flat-top transmission and a sharper passband, compared to the case where a single resonator is used as a filter. While the center frequency of the filter is determined mainly by the total length of the resonators, $L_{\text {res }}$, varying the inter-resonator ground plane width $G$ influences the frequency splitting between the symmetric- and anti-symmetric modes in the coupled resonators, as shown in Fig. 3(b). The airbridges across the filters are required for suppressing the slotline mode, which are observed to create an additional transmission peak when the bridges are absent. In practice, superconducting airbridges can readily be made across $\mathrm{NbTiN}$ groundplanes [10]. The leakage from the readout line to the signal line through the filter and the MKID at resonance has been calculated to be $<-50 \mathrm{~dB}$.

By using the scattering parameters calculated for individual filters, and connecting the signal lines via a modeled transmission line, we construct a 20-port network model that represents a filterbank with 18 filters. In order to enhance calculation speed, we use a MSL design for this particular analysys. Ports 1 and 2 represent the ports connected to the antenna and absorber, respectively, whereas ports 3-20 represent the outputs to the MKIDs. The transmission in the ideal case, in which there are no fabrication errors, is presented in Fig. 4(a). A resolving power of 1000 is achieved, with a flat-top bandpass of $\sim 80 \%$. However, in reality, errors in fabrication could deteriorate the spectral sampling. We assume a process in which the patterns are written directly using an electron-beam. Because the neighboring filters have a designed length difference of $50 \mathrm{~nm}$ at minimum, the pattern definition accuracy should be significantly better than this. This is at the border of what modern e-beam pattern generators can handle, and it is a capability that is usually not specified explicitly. For example, our VISTEC EBPG 5000+ is specified to be have a 'main field stability better than $40 \mathrm{~nm}$ over a 30 minute period,' whereas one filter can be written within a minute, using a beam that is $20 \mathrm{~nm}$ in diameter, and moved in steps of $5 \mathrm{~nm}$. 

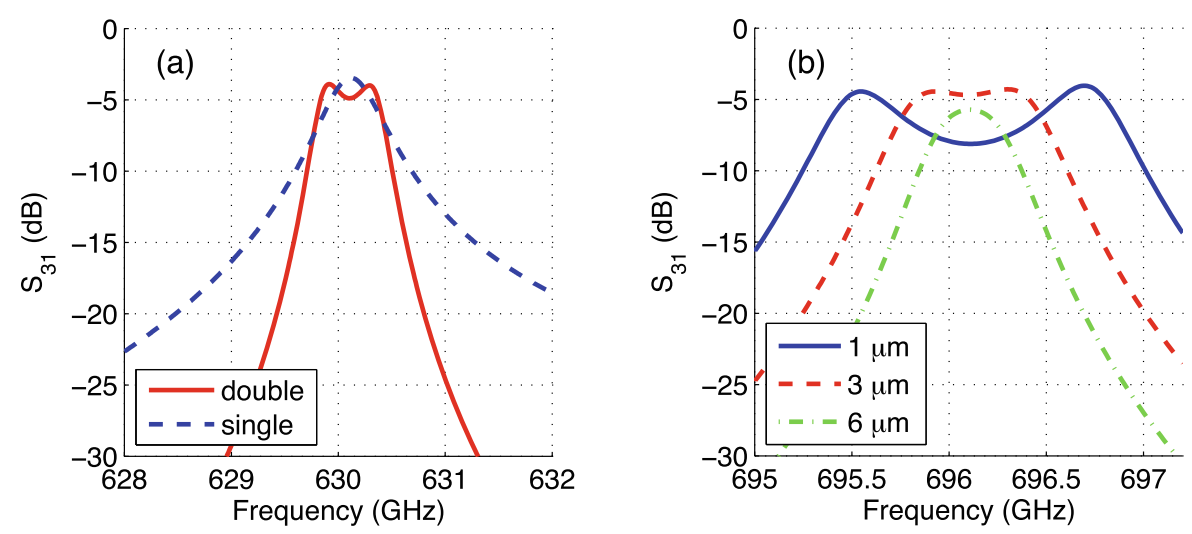

Fig. 3 (Color online) (a) Calculated transmission $S_{31}$ of filters with 1 (dashed) and 2 (solid) halfwave resonators. The geometry of the simulated model is described in Fig. 2, with $G=3 \mu \mathrm{m}$ and $L_{\mathrm{c}}=15 \mu \mathrm{m}$. $L_{\text {res }}$ has been adjusted so that the center frequency is equal in both cases. (b) Transmission $S_{31}$ of a filter with 2 coupled resonators with varying gap width $G$

(a) $\sigma=0 \mathrm{~nm}, \mu=0 \mathrm{~nm}$

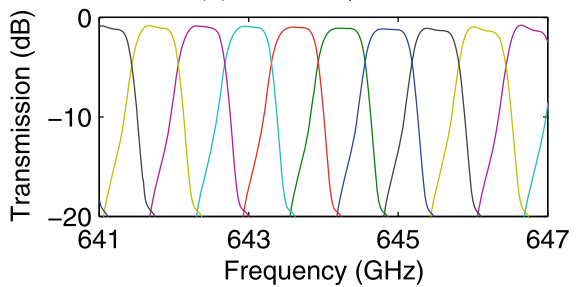

(b) $\sigma=0 \mathrm{~nm}, \mu=500 \mathrm{~nm}$

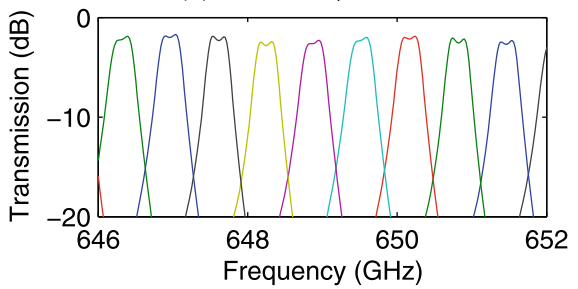

(c) $\sigma=10 \mathrm{~nm}, \mu=0 \mathrm{~nm}$

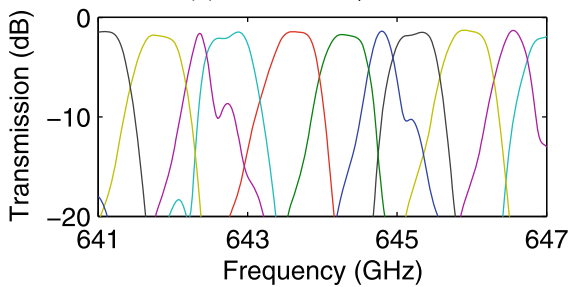

(d) $\sigma=20 \mathrm{~nm}, \mu=0 \mathrm{~nm}$

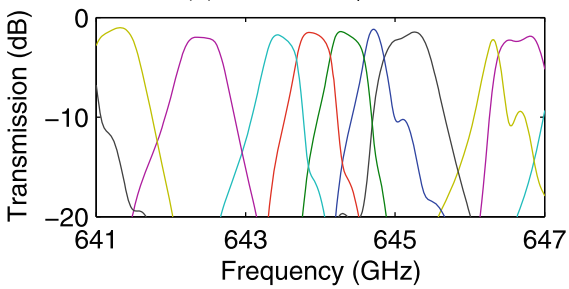

Fig. 4 (Color online) Simulated filter transmission of an 18-channel filterbank. The center $6 \mathrm{GHz}$ is taken as a representative. Each curve represents the transmission to a different filter. Each subfigure assumes a standard deviation of $\sigma \mathrm{nm}$ and an overall drift of $\mu \mathrm{nm}$ in the lateral etching of the metal

One of the fabrication processes that is especially difficult to control is the lateral etching of the metal. Over/under exposure of the resist, variations in the temperature, and chemical concentration, during the development of the resist, and the plasma conditions during the reactive ion etching, will all introduce an error in the area of the metal that is removed. Here we include a channel-to-channel error in lateral etching into our numerical model by shrinking/expanding the size of the metal sheets everywhere, with a standard deviation of $\sigma \mathrm{nm}$ and a common drift of $\mu \mathrm{nm}$. Positive and negative values correspond to a shrinkage and expansion at the metal edges, 
respectively. The results are presented in Fig. 4(b)-(d). With $\sigma=20 \mathrm{~nm}$, there are already 'empty slots' in the sampling. On the other hand, a common drift as large as $\mu=500 \mathrm{~nm}$ alone is not detrimental. Similar conclusions can be made with other sources of errors, such as the metal thickness variation: Random errors are much more harmful than a common drift. On top of this, there can be inter-filter crosstalk [11], which could further randomize the filter positions. How small the scatter can be made is an issue to be addressed both by simulation and experiment. Using multiple pixels would be a possible way to recover the whole spectral information, when each pixel has an incomplete sampling.

\section{Conclusion}

We have introduced the concept of an IFB, as an attractive solution for realizing a compact and broadband submillimeter wave spectrograph with 2D spatial sampling. Numerical simulation shows that, assuming an idealized situation in which frequency scattering is absent, the coupling efficiency to a filter reaches $80 \%$. Figure 1 (b) is an example layout of a 9-pixel spectrograph integrated onto a 4-inch wafer. The device is designed to cover $320-475 \mathrm{GHz}$ and $600-950 \mathrm{GHz}$ with a resolving power of 1000 . Issues such as fabrication accuracy, stray light coupling and crosstalk, should be further investigated both by simulation and by experiment, in order to find out whether the IFB is a viable route towards the realization of envisioned next-generation spectrographs, such as DESHIMA.

Acknowledgements We would like to thank E.F.C. Driessen for his inspiring comments. AE is financially supported by NWO (Veni grant 639.041.023) and JSPS Fellowship for Research Abroad. TMK likes to thank the W.M.Keck Institute for Space Sciences for partial support for his stay at California Institute of Technology, while this manuscript was being written.

Open Access This article is distributed under the terms of the Creative Commons Attribution Noncommercial License which permits any noncommercial use, distribution, and reproduction in any medium, provided the original author(s) and source are credited.

\section{References}

1. A. Amblard, A. Cooray, P. Serra, B. Altieri, V. Arumugam, H. Aussel, A. Blain, J. Bock, A. Boselli, V. Buat, and 64 coauthors. Nature 470, 510 (2011)

2. C.L. Carilli, R. Wang, Astron. J. 131, 2763 (2006)

3. G.J. Stacey, IEEE Trans. Terahertz Sci. Technol. 1, 241 (2011)

4. H. Inami, M. Bradford, J. Aguirre, L. Earle, B. Naylor, H. Matsuhara, J. Glenn, H. Nguyen, J.J. Bock, J. Zmuidzinas, Y. Ohyama, Proc. SPIE 7020, $70201 \mathrm{~T}$ (2008)

5. J.J.A. Baselmans, J. Low Temp. Phys. (2012). doi:10.1007/s10909-011-0448-8

6. J.A. Tauber, N.R. Erickson, Rev. Sci. Instrum. 62, 1288 (1991)

7. S.J.C. Yates, J.J.A. Baselmans, A. Endo, R.M.J. Janssen, L. Ferrari, A.M. Baryshev, Appl. Phys. Lett. 99, 073505 (2011)

8. R. Barends, N. Vercruyssen, A. Endo, P.J. de Visser, T. Zijlstra, T.M. Klapwijk, J.J.A. Baselmans, Appl. Phys. Lett. 97, 033507 (2010)

9. gSonnet Userfs Guide, Release 12,h. Sonnet Software Inc., North Syracuse, NY (2009)

10. Y.J.Y. Lankwarden, A. Endo, J. Baselmans, M. Bruijn, J. Low Temp. Phys. (2012) this issue

11. O. Noroozian, P.K. Day, B.H. Eom, H.G. Leduc, J. Zmuidzinas, IEEE Trans. Microw. Theory Tech. (submitted) 